Herz $2017 \cdot 42: 449-458$

DOI 10.1007/s00059-017-4578-x

Online publiziert:29. Mai 2017

(c) Der/die Autor(en) 2017. Dieser Artikel ist eine Open-Access-Publikation.

CrossMark

\author{
W. März ${ }^{1,2,3} \cdot$ T. B. Grammer ${ }^{4}$ G. Delgado' $\cdot$ M. E. Kleber ${ }^{1}$ \\ ${ }^{1}$ Medizinische Klinik V (Nephrologie, Hypertensiologie, Rheumatologie, Endokrinologie, Diabetologie), \\ Medizinische Fakultät Mannheim der Universität Heidelberg, Mannheim, Deutschland \\ ${ }^{2}$ Klinisches Institut für Medizinische und Chemische Labordiagnostik, Medizinische Universität Graz, Graz, \\ Österreich \\ ${ }^{3}$ Synlab Akademie, synlab Holding Deutschland GmbH, Mannheim, Deutschland \\ ${ }^{4}$ Institut für Public Health, Medizinische Fakultät Mannheim der Universität Heidelberg, Mannheim \\ Deutschland
}

\title{
Angeborene Störungen im Lipoproteinstoffwechsel
}

über 50 Jahren und bei Männern über 40 Jahren, bei Kindern, wenn in der Familie vorzeitige Atherosklerose oder Fettstoffwechselstörungen vorkommen, und jederzeit bei Patienten mit kardiovaskulären Risikofaktoren oder Erkrankungen [13, 57]. Das LDL-C wird heute entweder aus Gesamtcholesterin, Triglyzeriden und HDL-C nach Friedewald [20] errechnet oder „direkt“ bestimmt. Die „direkten“ Bestimmungen des LDLC sind der Friedewald-Formel kaum überlegen.

Sekundäre HLP sind weit häufiger als primäre HLP. Sie treten auf als Folge von Adipositas, Fehlernährung, Diabetes mellitus, exzessivem Alkoholkonsum, nephrotischem Syndrom, chronischem Nierenversagen, Hypothyreose und unter der Einnahme von Medikamenten (Kontrazeptiva, Betablocker, Diuretika, Glukokortikoide, Retinoide; - Tab. 1 und 2). Bei sekundären HLP ist die auslösende Ursache zu behandeln. Persistiert die sekundäre HLP, ist sie wie eine primäre Form zu behandeln.

An angeborene Störungen des Fettstoffwechsels ist zu denken, wenn es sich um junge Patienten handelt, die Konzentrationen des LDL-C über $190 \mathrm{mg} / \mathrm{dl}$ (4,9 mmol/l) und/oder der Triglyzeride über $200 \mathrm{mg} / \mathrm{dl}$ (2,3 mmol/l) liegen, eine sekundäre HLP ausgeschlossen werden kann oder wenn sich bei Angehörigen ebenfalls erhöhte Lipidkonzentrationen oder frühzeitige Herzinfarkte finden. Für eine primäre HLP sprechen auch das Auftreten von Xanthelasmen (wenig spezifisch), Arcus lipoides, oder Xanthomen sowie abdominelle Beschwerden (Pankreasaffektionen bei Chylomikronämie).

\section{Störungen im Cholesterinstoff- wechsel}

\section{Autosomal-dominante familiäre Hypercholesterinämie}

Etwa jeder 250. Mensch in Deutschland leidet an einer autosomal-dominanten FH [37]. Die FH geht mit einer Erhöhung von LDL im Blut und meist fortschreitender Atherosklerose einher - auch wenn keine weiteren kardiovaskulären Risikofaktoren vorliegen.

Die FH ist definiert als eine Störung im Abbau der LDL. Bei 93 \% der Betroffenen liegen die verantwortlichen Mutationen im Gen des LDL-Rezeptors (LDL-R; [27, $52,54])$, bei $5 \%$ findet man Mutationen der rezeptorbindenden Domäne des Apolipoproteins B-100 (APOB-100; [45, 47]) und bei $2 \%$ „Gain-of-function“-Mutationen von PCSK9 („proprotein convertase subtilisin/kexin type 9“; [1, 36, 68, 71-73]). PCSK9 ist eine Protease, die am zellulären Abbau von LDLR beteiligt ist. Wenn es aufgrund genetischer Veränderungen eine erhöhte Aktivität aufweist („gain of function“), werden mehr LDLR als normalerweise abgebaut. Neuerdings werden Mutationen von STAP1 (,signaltransducing adaptor protein $1^{\text {“) }}$ als Ursache einer FH diskutiert (• Tab. 3; [7]).

Zwischen 5 und $10 \%$ aller Koronarkranken unter 55 Jahren haben eine heterozygote $\mathrm{FH}$ (heFH; [3, 7, 77]). Das LDL-C ist mit $190-350 \mathrm{mg} / \mathrm{dl}$ Basisdiagnostik ist indiziert bei Frauen 
Tab. 1 Sekundäre Hyperlipoproteinämien

Grunderkrankung LDL $\begin{aligned} & \text { Trigly- } \\ & \text { zeride }\end{aligned} \quad$ HDL

Endokrinologie und Stoffwechsel

Hypothyreose $\quad \uparrow \uparrow \leftrightarrow, \uparrow \leftrightarrow$

Diabetes mellitus $\leftrightarrow \quad \uparrow \uparrow \quad \downarrow$

Akromegalie $\leftrightarrow \quad \uparrow \quad \uparrow$

Wachstumshor- $\quad \uparrow \quad \leftrightarrow \quad \downarrow$

monmangel

Hyperkortisolismus $\uparrow \quad \uparrow \quad \leftrightarrow$

Adipositas $\leftrightarrow \quad \uparrow \quad \downarrow$

Nierenerkrankungen

Nephrotisches $\quad \uparrow \quad \uparrow \quad \uparrow \quad \leftrightarrow$

Syndrom

Niereninsuffizienz $\leftrightarrow, \uparrow \quad \uparrow \uparrow \quad \leftrightarrow, \downarrow$

Nierentransplan- $\quad \uparrow \uparrow \quad \uparrow \quad \leftrightarrow$

tation

Lebererkrankungen

Cholestase $\quad \uparrow \quad \uparrow \quad \downarrow$

Hepatitis $\leftrightarrow, \uparrow \uparrow \uparrow \quad \downarrow$

Leberzirrhose $\quad \downarrow \quad \downarrow \quad \downarrow$

Sonstige Erkrankungen

Alkoholismus $\leftrightarrow \quad \uparrow \quad \uparrow$

Anorexie $\quad \uparrow \quad \leftrightarrow \quad \leftrightarrow, \downarrow$

$\leftrightarrow$ normal, $\uparrow$ erhöht, $\downarrow$ vermindert

$L D L$ "low-density lipoprotein", $H D L$ "highdensity lipoprotein"

(5,2-9,1 mmol/l) etwa 2-fach oder mehr erhöht. Bei Männern mit heFH beträgt das Risiko für eine koronare Herzkrankheit (KHK) $90 \%$ bis zum 60. Lebensjahr, bei Frauen $40 \%$. Das entspricht einer 12- bis 13-fachen Steigerung des Risikos $[3,77]$ und einer Verringerung der Lebenserwartung um etwa 15 Jahre. Die Hälfte der Verwandten ersten Grades ist ebenfalls betroffen. Patienten mit hoFH haben in der Regel ein LDL-C zwischen 600 und $1000 \mathrm{mg} / \mathrm{dl}$ (15,5 und 25,9 mmol/l). Die KHK manifestiert sich häufig im ersten Lebensjahrzehnt.

Die Indikation für eine genetische Untersuchung kann mit dem Punkteschema des Dutch Lipid Clinics Network [44, 78] gestellt werden (• Tab. 4, www.fhscore.eu). Ab einem Punktwert von 6 ist eine molekulargenetische Untersuchung sinnvoll. Der Nachweis der verantwortlichen genetischen Variante gelingt dann in mehr als $70 \%$ der Fälle [27].

Nur etwa ein Zehntel aller Patienten mit isolierter Erhöhung des LDL-C hat

Tab. 2 Weiterführende Diagnostik zum Ausschluss von Grunderkrankungen, die zu einer sekundären Dyslipoproteinämie führen ${ }^{2}$

\begin{tabular}{|c|c|}
\hline $\begin{array}{l}\text { Auszuschließende } \\
\text { Grunderkrankung }\end{array}$ & Geeignete (Labor-)Untersuchungen \\
\hline Hypothyreose & TSH (ggf. zusätzlich T3, T4) \\
\hline Diabetes mellitus & $\begin{array}{l}\text { Glukose (nüchtern, Tagesprofil, oraler Glukosetoleranztest, evtl. } \\
\mathrm{HbA}_{1 \mathrm{C}} \text { ) }\end{array}$ \\
\hline Nephrotisches Syndrom & Eiweiß im Urin, Eiweiß im Serum, Elektrophorese \\
\hline Niereninsuffizienz & Kreatinin, Harnstoff \\
\hline Cholestase & Y-GT, Bilirubin, alkalische Phosphatase \\
\hline Hepatitis & ALAT, ASAT (evtl. Hepatitisserologie) \\
\hline Leberzirrhose & $\begin{array}{l}\text { ALAT, ASAT, Pseudocholinesterase, Eiweiß im Serum, Elektrophorese, } \\
\text { Tromboplastinzeit nach Quick }\end{array}$ \\
\hline Chron. Alkoholabusus & Anamnese, $\gamma$-GT, kohlenhydratdefizientes Transferrin \\
\hline Anorexie & Anamnese, Gewicht \\
\hline \multicolumn{2}{|c|}{$\begin{array}{l}\text { aBei entsprechenden Hinweisen auch an Akromegalie, Wachstumshormon-Defizienz, Hyperkortiso- } \\
\text { lismus, akute Porphyrie usw. denken } \\
T S H \text { thyreoideastimulierendes Hormon, ALAT Alanin-Aminotransferase, ASAT Aspartat-Aminotrans- } \\
\text { ferase, } \gamma \text {-GT Gammaglutamyltranspeptidase }\end{array}$} \\
\hline
\end{tabular}

tatsächlich eine FH. Differenzialdiagnosen sind:

- polygene Hypercholesterinämie,

- autosomal-rezessive Hypercholesterinämie $(\mathrm{ARH})$,

- FKHL (• Abb. 1),

- sekundäre Hypercholesterinämien (Hypothyreose).

Die klinische Bedeutung des Mutationsnachweises belegt eine Studie von Khera und Kollegen [36], die gezeigt haben, dass der Nachweis einer sicher pathogenen Mutation in den Genen $L D L R, A P O B$ oder PCSK9 ein von LDL-C unabhängiger, kardiovaskulärer Risikofaktor ist [32, 36].

Weitere Gründe für die molekulare Diagnostik bei FH [37]:

- Die molekulare Diagnose verbessert die Therapietreue [75].

- Nach Identifizierung des genetischen Defekts in einer Familie können $\mathrm{Mu}$ tationsträger unter den Angehörigen identifiziert werden (Kaskadenscreening; [22, 72]).

- Bei Patienten mit gesicherter FH wird das globale kardiovaskuläre Risiko mit Risikoalgorithmen unterschätzt. Deshalb werden asymptomatische Personen mit FH auch ohne weitere Risikofaktoren als Patienten mit hohem Risiko eingestuft $[13,57]$.

- Aktuelle Leitlinien [57, 59] empfehlen bei FH einen Zielwert für LDL-
C von $100 \mathrm{mg} / \mathrm{dl}$ (2,6 mmol/l), bei KHK oder weiteren Risikofaktoren von $70 \mathrm{mg} / \mathrm{dl}$ (1,8 mmol/l; Klasse IIa, Evidenzgrad C). Durch Änderungen des Lebensstils und konventionelle Medikamente kann das LDL-C bei $\mathrm{FH}$ oft nicht ausreichend gesenkt werden. Aufgrund der Anlage III der Arzneimittel-Richtlinie (AMRL) sind die PCSK9-Antikörper Alirocumab und Evolocumab bei "gesicherter familiärer heterozygoter Hypercholesterinämie unter Berücksichtigung des Gesamtrisikos familiärer Belastung“ erstattungsfähig $[10,11]$. Die Dokumentation einer FH mit genetischen Methoden eignet sich zur objektiven Feststellung eines „hohen Gesamtrisikos familiärer Belastung" und kann damit die Indikation für eine Behandlung mit PCSK9-Antikörpern absichern.

\section{Autosomal-rezessive Hyper- cholesterinämie}

Die bisher bekannten Patienten mit ARH bieten klinisch das Bild einer homozygoten FH, wobei Eltern und Großeltern gewöhnlich normolipidämisch sind. Die Erkrankung wird durch Mutationen des LDL-R-Adapterproteins LDLRAP1 verursacht [25]. 


\section{Polygene Hypercholesterinämie}

Nicht bei allen Patienten, die aufgrund der klinischen Kriterien eine mögliche oder wahrscheinliche $\mathrm{FH}$ haben, lassen sich Mutationen der typischen Gene für FH nachweisen [14, 17, 23, 40].

Gelingt der Nachweis von Mutationen in den Genen $L D L R, A P O B$ oder PCSK9 bei Patienten mit den klinischen Merkmalen einer FH nicht, handelt es sich nicht um eine autosomal-dominante $\mathrm{FH}$, sondern in $90 \%$ der Fälle um eine polygene Hypercholesterinämie [22, 71, 72]. Sie entsteht durch Zusammenwirken von Mutationen oder Polymorphismen (Allelfrequenzen $>1 \%$ ), die für sich allein genommen das LDL-C nur wenig erhöhen, aber in der Summe einen großen Effekt haben können (• Abb. 1; [21, 71, 72]).

\section{Familiäre kombinierte Hyperlipoproteinämie}

Die FKHL ist mit einer Prävalenz von 1:100 die häufigste Form der primären HLP. Rund $10 \%$ der Patienten mit Myokardinfarkt haben eine FKHL. Bei der FKHL sind LDL-C und/oder Triglyzeride erhöht. Xanthome machen die Diagnose unwahrscheinlich. Die Pathogenese ist unklar; vielleicht spielt eine erhöhte Produktion von VLDL (,very lowdensity lipoprotein“) eine Rolle. Diese kann zur Hypertriglyzeridämie führen, bei Patienten mit effizienterer Lipolyse stehen hingegen erhöhte LDL-Konzentrationen im Vordergrund. Familienmitglieder mit Hypertriglyzeridämie haben vermutlich ein genauso hohes Risiko für KHK wie diejenigen mit Hypercholesterinämie. Die Vererbung ist am ehesten polygen, indem Mutationen oder Polymorphismen, die LDL-C und Triglyzeride erhöhen, zusammenkommen ([8, 29, 34, 41]; • Abb. 2).

\section{Abetalipoproteinämie, Hypobetalipoproteinämie und Anderson-Erkrankung}

Die Abetalipoproteinämie ist eine autosomal-rezessive Erkrankung, bei der VLDL und LDL praktisch vollständig fehlen. Klinisch findet man:

Herz 2017 · 42:449-458 DOI 10.1007/s00059-017-4578-x

(c) Der/die Autor(en) 2017. Dieser Artikel ist eine Open-Access-Publikation.

\section{W. März · T. B. Grammer · G. Delgado · M. E. Kleber}

\section{Angeborene Störungen im Lipoproteinstoffwechsel}

\section{Zusammenfassung}

Die angeborenen Störungen des Fettstoffwechsels werden durch eine breite Palette von Varianten in Genen für Rezeptoren, Apolipoproteine, Enzyme, Transferfaktoren und zelluläre Cholesterintransporter verursacht. Klinisch die größte Bedeutung haben die autosomal-dominante familiäre Hypercholesterinämie (FH) und die familiäre kombinierte Hyperlipoproteinämie (FKHL). Die FH hat eine Prävalenz von 1:250. Sie ist auf Mutationen des $\operatorname{LDL}($ „low-density lipoprotein")-Rezeptors (LDLR), seltener auf Mutationen von Apolipoprotein $B$ (APOB), PCSK9 („proprotein convertase subtilisin/kexin type $9^{\prime \prime}$ ) oder STAP-1 (,signal transducing adaptor family member $1^{\prime \prime}$ ) zurückzuführen. Die FH führt meist zu frühzeitiger Atherosklerose. Die Diagnose kann nur molekulargenetisch sicher gestellt werden. Der Nachweis von Mutationen an LDLR, APOB oder PCSK9 ist unabhängig vom Serumwert für LDL-Cholesterin ein Indikator für extrem hohes kardiovaskuläres Risiko. Die FKHL ist ebenfalls häufig (1:100) und kommt bei etwa $10 \%$ der Patienten mit frühem Myokardinfarkt vor. Sie entsteht durch Kombinationen von häufigen genetischen Varianten mit Wirkungen auf Triglyzeride und LDL-Cholesterin. Weitere monogene Hyperlipoproteinämien (HLP) betreffen den Abbau der Chylomikronen (familiäre Chylomikronämie) oder der "remnants" triglyzeridreicher Lipoproteine (Typ-IIIHyperlipoproteinämie). Im Stoffwechsel der HDL sind viele erbliche Störungen bekannt. Die atherogene Wirkung dieser Defekte ist unterschiedlich. Aktuell werden Sequenzierungsmethoden der zweiten Generation angewendet, um die relevanten Gene simultan zu sequenzieren. Diese Vorgehensweise liefert kostenneutral auch weitere Informationen wie genetisches Atheroskleroserisiko und Prädisposition zur Statinunverträglichkeit.

\section{Schlüsselwörter}

Lipoproteinstoffwechsel · Familiäre Hypercholesterinämie · Familiäre kombinierte Hyperlipoproteinämie · Atherosklerose · Next Generation Sequencing

\section{Congenital disorders of lipoprotein metabolism}

\section{Abstract}

Congenital disorders of lipid metabolism are caused by a wide range of variants of the genes for receptors, apolipoproteins, enzymes, transfer factors, and cellular cholesterol transporters. Clinically most relevant are autosomal dominant familial hypercholesterolemia $(\mathrm{FH})$ and familial combined hyperlipoproteinemia ( $\mathrm{FCHL}$ ). FH has a prevalence of 1:250. It is due to mutations of the low density lipoprotein (LDL) receptor, less often to mutations of the apolipoprotein $B(A P O B)$, the proprotein convertase subtilisin/kexin type 9 (PCSK9), or the signal transducing adapter family member 1 (STAP1). FH often leads to early atherosclerosis. Its diagnosis can definitely be made only by molecular genetic testing. The detection of mutations of the LDLR, APOB, or PCSK9 is an indicator for extremely high cardiovascular risk, independently of the concentration of $\mathrm{LDL}$ cholesterol. FCHL is also common (1:100) and is seen in about $10 \%$ of patients with early myocardial infarction.
It is produced by combinations of frequent genetic variants affecting triglycerides and LDL cholesterol. Other monogenic hyperlipoproteinemias (HLP) affect the catabolism of chylomicrons (familial chylomicronemia) or of remnants of triglyceride-rich lipoproteins (type III hyperlipoproteinemia). Multiple hereditary disorders in HDL metabolism with a broad spectrum of clinical significance - are known. Currently, second generation sequencing methods are used to simultaneously analyze multiple disease-causing genes. This approach cost-neutrally provides additional information such as the genetic risk of atherosclerosis and predisposition to statin intolerance.

\section{Keywords}

Lipoprotein metabolism - Familial hypercholesterolemia · Familial combined hypolipoproteinemia $\cdot$ Atherosclerosis $\cdot$ Next generation sequencing 
Tab. 3 Genetische Diagnostik bei Verdacht auf angeborene Fettstoffwechselstörungen

\begin{tabular}{|c|c|c|}
\hline Erkrankung & Gene & Häufigkeit \\
\hline \multicolumn{3}{|c|}{ Störungen im Stoffwechsel des Cholesterins und der LDL } \\
\hline $\begin{array}{l}\text { Autosomal-dominante familiäre } \\
\text { Hypercholesterinämie (FH) }\end{array}$ & LDLR, APOB, PCSK9, STAP1 & $1: 250$ \\
\hline $\begin{array}{l}\text { Autosomal-rezessive Hypercho- } \\
\text { lesterinämie (ARH) }\end{array}$ & LDLRAP1 & Selten \\
\hline Polygene Hypercholesterinämie & $\begin{array}{l}12 \text { Polymorphismen in Genen mit Effekt auf } \\
\text { LDL-C }\end{array}$ & Etwa 1:150 \\
\hline Familiäre kombinierte HLP & $\begin{array}{l}12 \text { Polymorphismen in Genen mit Effekt auf } \\
\text { LDL-C, } 11 \text { Polymorphismen in Genen mit } \\
\text { Effekt auf Triglyzeride }\end{array}$ & Etwa 1:100 \\
\hline Abetalipoproteinämie & MTP & Selten \\
\hline $\begin{array}{l}\text { Hypobetalipoproteinämie, domi- } \\
\text { nant }\end{array}$ & PCSK9, APOE, ANGPTL3, APOB, NPC1L1 & Selten \\
\hline $\begin{array}{l}\text { Hypobetalipoproteinämie, rezes- } \\
\text { siv }\end{array}$ & SAR1B & Selten \\
\hline Familiäre Phytosterolämie & $A B C G 5, A B C G 8, N P C 1 L 1$ & Selten \\
\hline Zerebrotendinöse Xanthomatose & CYP27A & Selten \\
\hline Desmosterolose & DHCR24 & Selten \\
\hline \multicolumn{2}{|c|}{$\begin{array}{l}\text { Cholesterinester-Speicherkrankheit, LAL } \\
\text { Wolman-Erkrankung }\end{array}$} & Selten \\
\hline $\begin{array}{l}\text { Erhöhtes Lipoprotein (a) } \\
\text { (>20 mg/dl) }\end{array}$ & $L P A$ & $1: 5$ bis $1: 10$ \\
\hline \multicolumn{3}{|c|}{ Störungen vorwiegend im Stoffwechsel der "remnants" triglyzeridreicher Lipoproteine } \\
\hline Typ-III-Hyperlipoproteinämie & $\begin{array}{l}\text { APOE, } 11 \text { Polymorphismen in Genen mit } \\
\text { Effekt auf Triglyzeride }\end{array}$ & $1: 2000$ \\
\hline Mangel an hepatischer Lipase & $\begin{array}{l}\text { LIPC, } 11 \text { Polymorphismen in Genen mit Effekt } \\
\text { auf Triglyzeride }\end{array}$ & Selten \\
\hline \multicolumn{3}{|c|}{ Störungen vorwiegend im Stoffwechsel der triglyzeridreichen Lipoproteine } \\
\hline Familiäre Chylomikronämie & $L P L, A P O C 2, A P O A 5, L M F 1, G P I H B P 1$ & $1: 1.000 .000$ \\
\hline Polygene Hypertriglyzeridämie & $\begin{array}{l}11 \text { Polymorphismen in Genen mit Effekt auf } \\
\text { Triglyzeride }\end{array}$ & $1: 50$ \\
\hline Lipodystrophien & 21 Kandidatengene & Selten \\
\hline \multicolumn{3}{|c|}{ Störungen vorwiegend im Stoffwechsel der HDL } \\
\hline Apolipoprotein-A1-Mangel & APOA1 & Selten \\
\hline $\begin{array}{l}\text { Apolipoprotein-A1-assoziierte } \\
\text { Amyloidose }\end{array}$ & APOA1 & Selten \\
\hline „Tangier disease" & $A B C A 1$ & Selten \\
\hline LCAT-Mangel & $L C A T$ & Selten \\
\hline CETP-Mangel & CETP & Selten \\
\hline $\begin{array}{l}\text { Polygene Hypoalphalipoprote- } \\
\text { inämie }\end{array}$ & 21 Kandidatengene & - \\
\hline \multicolumn{3}{|l|}{ Risikoabschätzung, Pharmakokinetik } \\
\hline Genetisches Herzinfarktrisiko & 11 Polymorphismen in Risikogenen & - \\
\hline SAMS, Myopathien & 14 Kandidatengene & - \\
\hline \multicolumn{3}{|c|}{$\begin{array}{l}L D L \text { "low-density lipoprotein", } L D L-C L D L-C h o l e s t e r i n, ~ H L P \text { Hyperlipoproteinämie, } H D L \text { "high-den- } \\
\text { sity lipoprotein", LCAT Lecithin-Cholesterin-Acyltransferase, CETP Cholesterinestertransferprotein, } \\
\text { SAMS statinassoziierte Muskelsymptome }\end{array}$} \\
\hline
\end{tabular}

- Fettmalabsorption,

- Akanthozytose,

- spinozerebelläre Ataxie,

- periphere Neuropathie,

- Retinitis pigmentosa,

- Myopathie.

In einigen Familien wurden Defekte des Gens für das mikrosomale Triglyzeridtransferprotein (MTP) als Ursache identifiziert.

Im Gegensatz zur Abetalipoproteinämie wird die Hypobetalipoproteinämie [58] autosomal-kodominant übertragen. LDL-C und $\mathrm{APOB}$ sind bei Heterozygoten auf etwa ein Viertel vermindert; die klinischen Symptome sind gering (Bauchschmerzen, Akanthozytose, Neuropathie, Steatosis hepatis). Oft sind Mutationen des $A P O B$-Gens, die zur Synthese verkürzter Formen des Proteins führen, verantwortlich. „Loss-offunction"-Mutationen des Gens PCSK9 können auch Ursache einer kodominant vererbten Hypobetalipoproteinämie sein $[1,16]$. Sowohl bei der heterozygoten als auch bei der homozygoten Form sind die Betroffenen asymptomatisch [83]. Zwei verwandte Probanden mit kombinierter Hypolipidämie waren zusammengesetzt heterozygot für Mutationen des ANGPTL3 („angiopoietin-like 3 protein“, Inhibitor von Lipasen; [51]).

Bei der rezessiven Hypobetalipoproteinämie („chylomicron retention disease“, "Anderson's disease“) kommt es zur Fettmalabsorption infolge verminderter Freisetzung von Chylomikronen aus der intestinalen Mukosa. Verantwortlich für die Erkrankung sind Defekte der SAR1B („secretion associated Ras related GTPase 1B“; [35]).

\section{Familiäre Phytosterolämie}

Die Phytosterolämie ist eine autosomalrezessive Erkrankung [2, 4], die klinisch mit der $\mathrm{FH}$ verwechselt werden kann (Xanthome). Die Diagnose erfolgt durch Bestimmung der Pflanzensterole im Blut, deren Konzentrationen etwa 50-fach erhöht sind. Bei Kindern sind auch ausgeprägte Hypercholesterolämien bekannt.

Ursächlich sind Mutationen im ABCG5(„ATP binding cassette subfamily G member 5“)- oder ABCG8-Gen, 
Tab. 4 Punkteschema des Dutch Lipid Clinics Network $[44,78]$ zur klinischen Abschätzung der Wahrscheinlichkeit einer autosomal-dominanten familiären Hypercholesterinämie

\section{Kriterium}

Punkte

Familienanamnese

Verwandter 1. Grades mit frühzei- 1 tigen kardiovaskulären Ereignissen oder LDL-C >95. Perzentile

Verwandter 1. Grades mit Xan2 thomen oder Arcus lipoides oder Kinder $<18$ Jahren mit LDL-C $>95$. Perzentile

Anamnese

Frühzeitige KHK 2

Frühzeitige zerebrovaskuläre Erkrankung, periphere arterielle Verschlusskrankheit

Körperliche Untersuchung $\rightarrow$ Sehnenxanthome

Arcus lipoides (<45 Jahre)

LDL-C

$>328 \mathrm{mg} / \mathrm{dl}(8,5 \mathrm{mmol} / \mathrm{l}) \quad 8$

$250-328 \mathrm{mg} / \mathrm{dl}(6,5-8,5 \mathrm{mmol} / \mathrm{l}) \quad 5$

$193-259 \mathrm{mg} / \mathrm{dl}(5,0-6,0 \mathrm{mmol} / \mathrm{l}) \quad 3$

$155-193 \mathrm{mg} / \mathrm{dl}(4,0-6,0 \mathrm{mmol} / \mathrm{l}) \quad 1$

Genetik

Mutationsnachweis 8

LDL-C "Low-density liporotein"-Cholesterin, KHK koronare Herzkrankheit

8 und mehr Punkte: Diagnose sehr wahrscheinlich

6 und 7 Punkte: Diagnose wahrscheinlich

3 bis 5 Punkte: Diagnose möglich

Für die Berechnung des Scores werden die maximalen Punktzahlen aus jeder der Kategorien "Familienanamnese“, "Anamnese", "Körperliche Untersuchung", „LDL-C" und

"Genetik" zusammengezählt

Die Berechnung des Scores kann online vorgenommen werden, siehe auch http:// www.fhscore.eu

die 2 Komponenten eines Steroltransporters kodieren und v. a. im Darm und in der Leber exprimiert werden [4]. Aufgabe des ABCG5/G8-Kotransporters ist es, bereits resorbierte, aber unveresterte Sterole (und das sind v. a. pflanzliche Sterole) aus der Dünndarmzelle in das Darmlumen zurückzutransportieren. $\mathrm{Ob}$ Phytosterole eine höhere atherogene Potenz als Cholesterin aufweisen, ist offen $[26,66,67]$ : Häufig vorkommende Varianten des ABCG8 sind mit Phytosterolen und einem leicht erhöhten Herzinfarktri- siko assoziiert [74]. Nachdem diese aber auch mit Cholestanol (endogener Marker der Cholesterinresorption) korrelieren, könnte die beobachtete Beziehung zwischen ABCG8 und Koronarkrankheit [74] allgemein eine atherogene Wirkung einer hohen intestinalen Cholesterinresorption reflektieren [65].

Die Basistherapie bei Sitosterolämie besteht in einer an Pflanzensterolen armen Kost. Gallensäurebindende Harze und Ezetimibe senken Pflanzensterole.

\section{Zerebrotendinöse Xanthomatose}

Die Patienten haben ein moderat erhöhtes LDL-C und im Allgemeinen tendinöse Xanthome; verantwortlich sind Defekte im Gen CYP27A1 („sterol 27 hydroxylase“; [24]). Cholestanol ist stark erhöht, die Bildung der Gallensäure Chenodesoxycholsäure ist vermindert. Es entwickeln sich neurologische Ausfallerscheinungen bis hin $\mathrm{zu}$ schweren Ataxien. Die Behandlung erfolgt mit Chenodesoxycholsäure [49] und Statinen.

\section{Desmosterolose}

Die Patienten haben aufgrund von Defekten im DHCR24(„24-dehydrocholesterol reductase")-Gen hohe Plasmaspiegel von Desmosterol. Sie können bereits in der Kindheit erhebliche neurologische Funktionsstörungen entwickeln. Die Therapie erfolgt mit Statinen [5, 63].

\section{Mangel an lysosomaler saurer Lipase}

Beim Mangel an lysosomaler saurer Lipase („lysosomal acid lipase“, LIPA) kommt es zur Speicherung von Cholesterinestern und Triglyzeriden in vielen Organen [60, 79]. Die Erkrankung wird autosomal-rezessiv vererbt.

Es gibt 2 Verlaufsformen, nämlich die infantile (Wolman-Krankheit) und die adulte Form (Cholesterinester-Speicherkrankheit). Unbehandelt überleben Kinder mit Wolman-Krankheit kaum das erste Lebensjahr. Bei der adulten Verlaufsform findet man eine Hepatomegalie, erhöhte Leberenzyme, hohes LDL-C, hohe Triglyzeride und niedri- ges HDL-C. Auch beim LIPA-Gen sind häufig vorkommende Varianten mit einem leicht, aber genomweit signifikant erhöhten Herzinfarktrisiko assoziiert [80].

Die Lebenserwartung hängt von der Schwere des Enzymdefekts ab. Die Diagnose wird durch Messung der Enzymaktivität in Zellen des peripheren Blutes und in Fibroblasten und/oder den Nachweis von Mutationen im LIPA-Gen gestellt. Die klassische Behandlung wird neuerdings durch eine Langzeitenzymersatztherapie (Sebelipase alpha) ergänzt.

\section{Erhöhtes Lipoprotein(a)}

$\mathrm{Lp}$ (a) ist ein Komplex aus LDL und Apolipoprotein(a) [38]. Die Konzentration des $\mathrm{Lp}$ (a) ist genetisch determiniert und schwankt interindividuell in weiten Grenzen. Die Funktion des Lp(a) ist unklar. $L p(a)$ ist ein unabhängiger und kausaler Risikofaktor für die Entstehung von Atherosklerose [15, 18, 55].

Die Bestimmung Lp(a) ist v. a. bei Personen mit ,intermediärem“ kardiovaskulären Risiko aufgrund gängiger Prognosemodelle (SCORE, Framingham) oder bei Patienten mit frühzeitiger KHK und bei deren Angehörigen indiziert [13, 38, 55, 57]. Eine medikamentöse Senkung des $L p(a)$ ist schwierig. Bei schwerer, progredienter Koronarkrankheit und ansonsten gut eingestellten Lipiden kann die Elimination des Lp(a) mittels LDLApherese erwogen werden. In jedem Fall aber sollten bei hohem Lp(a) (>30 mg/dl) die Therapieziele für „,konventionelle“ $\mathrm{Ri}$ sikofaktoren (LDL-C, Blutdruck) strenger definiert werden.

\section{Störungen im Stoffwechsel der „remnants" triglyzeridreicher Lipoproteine}

\section{Typ-III-Hyperlipoproteinämie}

Klinische Charakteristika sind Exantheme der Handlinien, tuberöse oder tuberoeruptive Xanthome. Bei der familiären Typ-III-HLP akkumulieren die "remnants“ der triglyzeridreichen Lipoproteine im Plasma. Cholesterin und Triglyzeride sind auf Konzentrationen zwischen 300 und $600 \mathrm{mg} / \mathrm{dl}$ (3,4 und 
LDL-C erhöht, frühzeitige Koronarkrankheit, familiäre Prädisposition

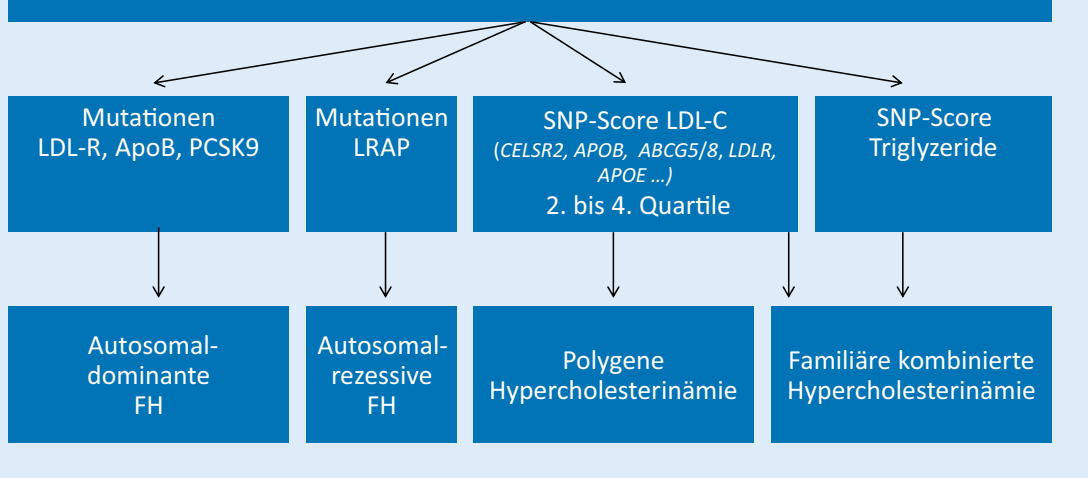

Abb. 1 ॥ Systematik genetisch bedingter Erhöhungen von „Low-density-lipoprotein“-Cholesterin ( $L D L-C)$ : Die autosomal-dominante familiäre Hypercholesterinämie $(F H)$ ist eine Störung im Abbau der LDL als Folge von Mutationen in den Genen für den LDL-Rezeptor (LDLR), Apolipoprotein B (APOB) und PCSK9 („proprotein convertase subtilisin/kexin type 9"). Die sehr seltene autosomal-rezessiveFH ist auf Mutationen im Gen des LDLR-Adapterproteins (LRAP) zurückzuführen [25]. Gelingt bei klinischem Verdacht aufFH und nach Ausschluss einer sekundären Erhöhung des LDL-C der Nachweis einer Mutation in diesen Genen nicht, handelt es sich mit hoher Wahrscheinlichkeit um eine polygene Hypercholesterinämie [71,72]. Sie kommt durch Zusammentreffen genetischer Polymorphismen mit Einfluss auf das LDL-C zustande. Die familiäre kombinierte Hyperlipoproteinämie (FKHL) ist ebenfalls häufig (1:100) und kommt bei bis zu $10 \%$ der Patienten mit frühem Myokardinfarkt vor. In den betroffenen Familien trifft man unterschiedliche Lipoproteinphänotypen an; LDL-C und/oder Triglyzeride sind erhöht. Die FKHL ist polygen vererbt und entsteht durch variable Kombinationen von SNPs (,single nucleotide polymorphisms") mit Wirkungen auf Triglyzeride und LDL-C ([71]; CELSR2 "cadherin EGF LAG seven-pass G-type receptor 2", ABCG5/8 „ATP-binding cassette sub-family G member 5/8“, APOE Apolipoprotein E)

6,8 mmol/l) erhöht. Die Lipoproteinelektrophorese zeigt eine breite $\beta$-Bande. Das LDL-C ist typischerweise niedrig. Die Störung manifestiert sich etwa nach dem 20. Lebensjahr. Patienten mit TypIII-HLP haben ein deutlich erhöhtes Atheroskleroserisiko [19].

Bei mehr als $90 \%$ der Patienten ist der APOE-Phäno- bzw. -Genotyp 2/2. Aber höchstens jeder 20. APOE2/2-Homozygote entwickelt eine Typ-III-HLP. Man nimmt an, dass die Manifestation durch zusätzliche, krankheitsauslösende Faktoren exogener (Alter, Adipositas, Insulinresistenz, Hypothyreose) und anderer genetischer Faktoren (siehe Abschnitt „Polygene Hypertriglyzeridämie“) gefördert wird.

Patienten mit rezessiver Typ-III-HLP sprechen gut auf eine lipidmodifizierte Diät an. Die Pharmakotherapie erfolgt in erster Linie mit Fibraten, auch Statine können versucht werden.

\section{Mangel an hepatischer Lipase}

Es sind nur wenige Fälle von familiärem Mangel an hepatischer Lipase (LIPC) be- kannt. Cholesterin und Triglyzeride sind erhöht. Eruptive und palmare Xanthome können vorkommen. Das Lipoproteinmuster hat Ähnlichkeit mit dem der Typ-III-HLP.

\section{Störungen vorwiegend im Stoff- wechsel der triglyzeridreichen Lipoproteine}

\section{Familiäre Chylomikronämie}

Die Chylomikronämie ist eine seltene, autosomal-rezessiv vererbte Störung im Abbau der triglyzeridreichen Lipoproteine. Im Nüchternplasma der Patienten findet man Chylomikronen, die Triglyzeride sind erhöht. Meist wird die Diagnose im Kindesalter aufgrund rezidivierender Pankreatitiden, eruptiver Xanthome, Hepatosplenomegalie und einer Lipaemia retinalis gestellt.

Ursachen sind Mutationen der Lipoproteinlipase (LPL) sowie von APOC2 (Kofaktor der LPL; [53]), GPIHBP1 („glycosylphosphatidylinositol-anchored high density lipoprotein-binding protein 1“) [81], APOA5 (Kofaktor der
LPL) und LMF1 („lipase maturation factor 1"). Heterozygote Träger von $\mathrm{Mu}$ tationen der LPL haben verminderte Enzymaktivitäten und leicht erhöhte Triglyzeride.

Die Therapie ist diätetisch. Maßnahmen bei akutem Chylomikronämiesyndrom umfassen

- absolute Nahrungskarenz,

- hypokalorische Infusionstherapie,

- Ausschalten der Auslöser (Alkohol, Östrogene),

- 5000 IE Heparin pro 12 h. Plasmaaustausch.

Eine Gentherapie zur Substitution der LPL (Alipogene tiparvovec) ist in Europa zugelassen.

\section{Polygene Hypertriglyzeridämie}

Bei Erwachsenen ist die Hypertriglyzeridämie häufig Folge genetischer Polymorphismen und seltener Varianten in Genen des Triglyzeridstoffwechsels, darunter APOA5, LPL, APOC3, ANGPTL4, $A P O B, G C K R$ („glucokinase regulator") und MLXIPL (,MLX interacting proteinlike“; [8, 29, 30, 34, 41, 69]). Die Triglyzeridkonzentrationen liegen zwischen 200 und $500 \mathrm{mg} / \mathrm{dl}(2,3$ und $5,7 \mathrm{mmol} / \mathrm{l})$. LDL-C und HDL-C sind niedrig. Die individuelle Ausprägung der Stoffwechselstörung wird durch Geschlecht, Alter, Ernährung, Insulinresistenz, Steatosis hepatis, Alkohol, Nierenfunktion oder Einnahme von Hormonen (Östrogene erhöhen die Triglyzeride) moduliert. Häufige Varianten in den Genen $L P L$, APOA5, APOC 3 und ANGPTL4 sind auch mit einem leicht, aber genomweit signifikant erhöhten Herzinfarktrisiko assoziiert [69].

Von der FKHL wird die Störung dadurch abgegrenzt, dass in den betroffenen Familien Erhöhungen des LDLC nicht vorkommen. Das Atheroskleroserisiko ist erhöht. Die Behandlung erfolgt diätetisch und umfasst Alkoholrestriktion und den Verzicht auf Östrogene. Als Medikamente kommen Omega3-Fettsäuren oder Fibrate in Betracht. 


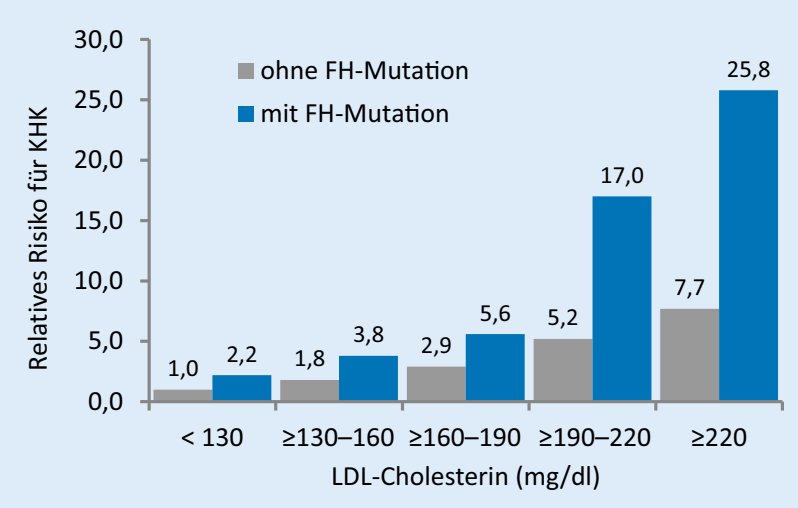

Abb. 2 ム Nachweis von FH(familiäre Hypercholesterinämie)-Mutationen und Koronarrisiko: Von 20.485 Personen ohne koronare Herzkrankheit (KHK) hatten 1386 (6,7 \%) ein LDL-C („Low-density-lipoprotein"-Cholesterin) über $190 \mathrm{mg} / \mathrm{dl}$ (4,9 mmol/l); 24 dieser Personen hatten Mutationen in den Genen $L D L R, A P O B$ oder PCSK9. Im Vergleich zu Personen mit LDL-C-Werten unter $130 \mathrm{mg} / \mathrm{dl}(3,4 \mathrm{mmol} / \mathrm{l})$ ohne Mutation hatten Personen mit LDL-C-Werten über $190 \mathrm{mg} / \mathrm{dl}$ (Mittelwert $203 \mathrm{mg} / \mathrm{dl}$ ) ein 6-fach erhöhtes Risiko für KHK. Bei LDL-C-Werten über $190 \mathrm{mg} / \mathrm{dl}$ (Mittelwert $205 \mathrm{mg} / \mathrm{dl}$ ) und nachgewiesener FH-Mutation war hingegen das Risiko 22-fach erhöht. Auch bei LDL-C-Konzentrationen unter $190 \mathrm{mg} / \mathrm{dl}$ erhöhte das Vorliegen pathogener Mutationen das Risiko etwa 2-fach [36]

\section{Lipodystrophiesyndrome}

Die genetisch heterogenen Lipodystrophien können mit ausgeprägten Erhöhungen der Triglyzeride bis hin zur Chylomikronämie und Entwicklung einer Pankreatitis einhergehen. Ihr gemeinsames Merkmal ist ein Mangel an Fettgewebe, der generalisiert oder partiell auftritt und genetisch bedingt oder erworben sein kann. Lipodystrophiesyndrome sind häufig mit hormonellen und metabolischen Störungen und Komorbiditäten assoziiert, die von Subtyp, Ausmaß des Fettabbaus, Alter und Geschlecht abhängen [9]. Die genetische Diagnostik und Differenzierung bedienen sich der simultanen Sequenzierung der 21 bekannten Kandidatengene.

\section{Dyslipoproteinämien mit niedriger Konzentration der HDL}

Niedriges HDL-C findet man oft gemeinsam mit hohen Triglyzeriden, auch bei primären HLP wie der familiären Chylomikronämie, der familiären Hypertriglyzeridämie und der FKHL. Es gilt heute als fraglich, ob eine niedrige Konzentration der HDL ursächlich für Atherosklerose ist. In jedem Fall ist HDL-C aber ein Marker für ein erhöhtes kardiovas- kuläres Risiko und das Vorliegen direkt proatherogener Lipoproteine [46].

Monogene, rezessive HDL-Mangelerkrankungen sind selten. Klinische Symptome des autosomal-rezessiven, kompletten LCAT(Lecithin-CholesterinAcyltransferase)-Mangels sind Hornhauttrübung, Anämie und Proteinurie. Das Atheroskleroserisiko von Mutationsträgern ist kaum erhöht [12]. Bei einigen Patienten wird der Verlauf der Erkrankung von der sich entwickelnden Niereninsuffizienz bestimmt. Diagnostisch verwertbar ist ein abnorm niedriges Verhältnis von Cholesterinestern $\mathrm{zu}$ freiem Cholesterin. Als Fischaugenkrankheit bezeichnet man eine partielle LCAT-Defizienz (verminderte LCATAktivität nur gegenüber exogenen Lipoproteinsubstraten). HDL-C ist auf etwa $10 \%$ der Norm vermindert, das Verhältnis von Cholesterinestern zu freiem Cholesterin ist leicht reduziert.

Die autosomal-rezessive Tangier-Erkrankung ist klinisch durch große, gelb gefärbte Tonsillen, Hepatosplenomegalie, periphere Neuropathie und fast nicht nachweisbares HDL-C gekennzeichnet. Der Abtransport von Cholesterin aus Zellen ist als Folge von Mutationen im Gen des ABCA1(„ATP-binding cassette A1")-Transporters gestört [6, 62]. Patienten mit Tangier-Krankheit haben oft auch ein niedriges LDL-C, was den atherogenen Effekt des niedrigen HDLC abschwächen könnte [64].

Homozygote "Nonsense“-Mutationen im APOA1-Gen wurden als Ursache von HDL-Mangel bei Patienten mit ausgeprägter Xanthomatose und frühzeitiger Atherosklerose gefunden.

Heterozygote Mutationen in den Genen für APOA1, ABCA1 oder LCAT können zu niedrigem HDL-C führen [64]. Ob sie auch das Atheroskleroserisiko erhöhten, ist unklar [50]. Einige Mutationen im APOA1 wurden mit erhöhtem Herzinfarktrisiko assoziiert [31], eine andere - APOA1(Milano) - dagegen mit einem erniedrigten Risiko. Bestimmte Mutationen im APOA1-Gen verursachen eine familiäre Amyloidose.

Die meisten Patienten mit einem Mangel an Cholesterinestertransferpotein (CETP) haben sehr hohe Konzentrationen an HDL-C. In klinischen Studien senkten 3 Hemmstoffe des CETP die Rate kardiovaskulärer Ereignisse nicht, ein Hemmstoff (Anacetrapib) wird noch untersucht. Wir haben bei niedrigen CETP-Konzentrationen im Blut trotz hohen HDL-C sogar eine leicht erhöhte kardiovaskuläre Mortalität beobachtet [61].

Erst neuerdings wurden Mutationen des SR-BI („scavenger receptor B1“) beschrieben, die mit deutlich erhöhtem HDL-C und erhöhtem kardiovaskulären Risiko einhergehen [43, 76, 82]. Ein hohes HDL-C schützt daher nicht in jedem Fall vor Atherosklerose.

\section{Statinassoziierte Muskel- beschwerden (SAMS)}

Muskelsymptome sind die klinisch bedeutsamste Nebenwirkung der Therapie mit Statinen und führen oft dazu, dass die Behandlung abgesetzt wird $[39,48,70]$.In randomisierten klinischen Studien liegen die Häufigkeiten von Muskelbeschwerden in den Placebo- und Verumgruppen eng beieinander; in Registerstudien werden die Inzidenzraten mit $10-30 \%$ der Behandelten angegeben.

Genomweite Analysen haben eine enge Assoziation zwischen einem Polymorphismus des SLCO1B1 („solute carrier organic anion transporter $1 \mathrm{~B} 1$ ", c.521T>C, p.V174A) und der Häufigkeit 
von SAMS unter Simvastatin festgestellt $[42,56]$. Heterozygote Träger der Genvariante haben ein 4,5-fach, homozygote ein 17-fach erhöhtes Risiko für eine Myopathie unter Simvastatin.

Die Einnahme von Statinen kann dazu führen, dass vorbestehende, milde Myopathien symptomatisch werden. Eine verzögerte Remission von SAMS nach Absetzen des Statins kann dabei hinweisend sein. Mögliche Grunderkrankungen sind u.a. Gykogenphosphorylasemangel, CPT(Carnitin-Palmitoyl-Transferase)-II-Mangel, Myoadenylatdesaminasemangel und maligne Hyperthermie [39, $48,70]$.

\section{Paneldiagnostik mit Sequenzierungsmethoden der zweiten Generation}

Seit vielen Jahren erfolgt die molekulargenetische Diagnostik von Fettstoffwechselstörungen mit der Sequenzierungsmethode nach Sanger. Da die Kapazität dieser Methode auch mit modernen Geräten begrenzt ist, mussten bislang anhand der klinischen Situation und der Lipidkonstellation diejenigen Gene für die Sequenzierung ausgewählt werden, in denen Mutationen am wahrscheinlichsten waren. Bei negativen Befunden wurden weitere Gene in die Suche eingeschlossen. Sequenzierungsmethoden der zweiten Generation („,next generation sequencing“, NGS) erlauben es nun, ohne wesentliche Mehrkosten simultan ganze Gruppen relevanter Gene zu sequenzieren $[28,33]$. Da nicht selten Defektvarianten in verschiedenen Genen in Kombination die Ausprägung des klinischen Phänotyps bestimmen, genetische Polymorphismen die Effekte schwerwiegender Mutationen modulieren und andererseits Mutationen an denselben Genen unterschiedliche Phänotypen verursachen, liefert die simultane Analyse mehrerer Gene umfassende differenzialdiagnostische Informationen.

\section{Fazit für die Praxis}

\section{- Angeborene Störungen des Lipo- proteinstoffwechsels beweisen die ursächliche Beteiligung erhöhter}

Cholesterinkonzentrationen an der Entstehung der Atherosklerose.

- In der Praxis ist es wichtig, die primären (d. $h$. genetisch bedingten) Fettstoffwechselstörungen von denjenigen zu trennen, die durch Wechselwirkung wenig penetranter genetischer Prädispositionen mit Lebensstilfaktoren oder durch definierte Grunderkrankungen entstehen.

- Die Fortschritte in der molekularen Diagnostik haben die Erkennung primärer Hyperlipoproteinämien wesentlich erleichtert.

- Sequenzierungsmethoden der zweiten Generation (,next generation sequencing") erlauben es, ohne wesentliche Mehrkosten simultan ganze Gruppen relevanter Gene zu sequenzieren.

\section{Korrespondenzadresse}

Prof. Dr. med. W. März

Synlab Akademie, synlab Holding Deutschland $\mathrm{GmbH}$

P5,7, 68167 Mannheim, Deutschland

winfried.maerz@synlab.com

Open access funding provided by Medical University of Graz.

\section{Einhaltung ethischer Richtlinien}

Interessenkonflikt. W. März erhält Unterstützung und Honorare von Siemens Diagnostics, Aegerion Pharmaceuticals, Amgen, AstraZeneca, Danone, Sanofi/Genzyme, Pfizer, BASF, Numares AG, Honorare von Berlin-Chemie, MSD, Sanofi, Synageva, Abbott Diagnostics; Beschäftigungsverhältnis: Synlab Holding Deutschland GmbH. T.B. Grammer, G. Delgado und M.E. Kleber geben an, dass kein Interessenkonflikt besteht.

Dieser Beitrag beinhaltet keine von den Autoren durchgeführten Studien an Menschen oder Tieren.

Open Access Dieser Artikel wird unter der Creative Commons Namensnennung 4.0 International Lizenz (http://creativecommons.org/licenses/by/4.0/deed. de) veröffentlicht, welche die Nutzung, Vervielfältigung, Bearbeitung, Verbreitung und Wiedergabe in jeglichem Medium und Format erlaubt, sofern Sie den/die ursprünglichen Autor(en) und die Quelle ordnungsgemäßnennen, einen Linkzur Creative Commons Lizenz beifügen und angeben, ob Änderungen vorgenommen wurden.

\section{Literatur}

1. Abifadel M, Varret M, Rabes JP et al (2003) Mutations in PCSK9 cause autosomal dominant hypercholesterolemia. Nat Genet 34:154-156

2. Beaty TH, Kwiterovich PO Jr., Khoury MJ et al (1986) Genetic analysis of plasma sitosterol, apoprotein $\mathrm{B}$, and lipoproteins in a large Amish pedigree with sitosterolemia. Am J Hum Genet 38:492-504

3. Benn M, Watts GF, Tybjaerg-Hansen A et al (2012) Familial hypercholesterolemia in the danish general population: prevalence, coronary artery disease, and cholesterol-lowering medication. JClin Endocrinol Metab 97:3956-3964

4. Berge KE, Tian H, GrafGA et al (2000) Accumulation of dietary cholesterol in sitosterolemia caused by mutations in adjacent $A B C$ transporters. Science 290:1771-1775

5. Bjorkhem I, Leoni V, Meaney S (2010) Genetic connections between neurological disorders and cholesterol metabolism. J Lipid Res 51:2489-2503

6. Bodzioch M, Orso E, Klucken J et al (1999) The gene encoding ATP-binding cassette transporter 1 is mutated in Tangier disease. Nat Genet 22:347-351

7. Braenne I, Kleinecke M, Reiz B et al (2016) Systematic analysis of variants related to familial hypercholesterolemia in families with premature myocardialinfarction. Eur JHum Genet 24:191-197

8. Brahm AJ, Hegele RA (2016) Combined hyperlipidemia: familial but not (usually) monogenic. Curr Opin Lipidol 27:131-140

9. Brown RJ, Araujo-Vilar D, Cheung PT et al (2016) The diagnosis and management of lipodystrophy syndromes: a multi-society practice guideline. JClin Endocrinol Metab 101:4500-4511

10. Bundesausschuss G (2016) Beschluss des Gemeinsamen Bundesausschusses über eine Änderung der Arzneimittel-Richtlinie (AM-RL): Anlage III Übersicht über Verordnungseinschränkungen und -ausschlüsse Alirocumab. 4. August 2016

11. Bundesausschuss G (2016) Beschluss des Gemeinsamen Bundesausschusses über eine Änderung der Arzneimittel-Richtlinie (AM-RL): Anlage III Übersicht über Verordnungseinschränkungen und -ausschlüsse Evolocumab. BAnz AT 12. Aug. 2016 B1

12. Calabresi L, Baldassarre D, Castelnuovo $S$ et al (2009) Functional lecithin: cholesterol acyltransferase is not required for efficient atheroprotection in humans. Circulation 120:628-635

13. Catapano AL, Graham I, De Backer G et al (2016) 2016 ESC/EAS Guidelines for the Management of Dyslipidaemias: The Task Force for the Management of Dyslipidaemias of the European Society of Cardiology (ESC) and European Atherosclerosis Society (EAS) Developed with the special contribution of the European Assocciation for Cardiovascular Prevention \& Rehabilitation (EACPR). Atherosclerosis 253:281-344

14. Civeira F, Ros E, Jarauta Eet al (2008) Comparison of genetic versus clinical diagnosis in familial hypercholesterolemia. Am J Cardiol 102:1187-1193.e1

15. Clarke R, Peden JF, Hopewell JC et al (2009) Genetic variants associated with $L p(a)$ lipoprotein level and coronary disease. N Engl J Med 361:2518-2528

16. Cohen JC, Boerwinkle E, Mosley TH et al (2006) Sequence variations in PCSK9, low LDL, and protection against coronary heart disease. N Engl J Med 354:1264-1272

17. Damgaard D, Larsen ML, Nissen PH et al (2005) The relationship of molecular genetic to clinical diagnosis of familial hypercholesterolemia in a Danish population. Atherosclerosis 180:155-160 
18. Erqou S, Kaptoge S, Perry PL et al (2009) Lipoprotein(a) concentration and the risk of coronary heart disease, stroke, and nonvascular mortality. JAMA 302:412-423

19. Feussner G, Feussner V, Hoffmann MM et al (1998) Molecular basis of type III hyperlipoproteinemia in Germany. Hum Mutat 11:417-423

20. Friedewald WT, Levy RI, Fredrickson DS (1972) Estimation of the concentration of low-density lipoprotein cholesterol in plasma, without use of the preparative ultracentrifuge. Clin Chem 18:499-502

21. Futema M, Plagnol V, Li Ket al (2014) Whole exome sequencing of familial hypercholesterolaemia patients negative for LDLR/APOB/PCSK9 mutations. J Med Genet 51:537-544

22. Futema M, Shah S, Cooper JA et al (2015) Refinement of variant selection for the LDL cholesterol genetic risk score in the diagnosis of the polygenic form of clinical familial hypercholesterolemia and replication in samples from 6 countries. Clin Chem 61:231-238

23. Futema M, Whittall RA, Kiley A et al (2013) Analysis of the frequency and spectrum of mutations recognised to cause familial hypercholesterolaemia in routine clinical practice in a UK specialist hospital lipid clinic. Atherosclerosis 229:161-168

24. Gallus GN, Dotti MT, Federico A (2006) Clinical and molecular diagnosis of cerebrotendinous xanthomatosis with a review of the mutations in the CYP27A1 gene. Neurol Sci 27:143-149

25. Garcia CK, Wilund K, Arca M et al (2001) Autosomal recessive hypercholesterolemia caused by mutations in a putative LDL receptor adaptor protein. Science 292:1394-1398

26. Genser B, Silbernagel G, De Backer G et al (2012) Plant sterols and cardiovascular disease: a systematic review and meta-analysis. Eur Heart 33:444-451

27. Grenkowitz T, Kassner U, Wuhle-Demuth $M$ et al (2016) Clinical characterization and mutation spectrum of German patients with familial hypercholesterolemia. Atherosclerosis 253:88-93

28. Hegele RA, Ban MR, Cao H et al (2015) Targeted next-generation sequencing in monogenic dyslipidemias. Curr Opin Lipidol 26:103-113

29. Hegele RA, Ban MR, Hsueh N et al (2009) A polygenic basis for four classical Fredrickson hyperlipoproteinemia phenotypes that are characterized by hypertriglyceridemia. Hum Mol Genet 18:4189-4194

30. Hegele RA, Ginsberg HN, Chapman MJ et al (2014) The polygenic nature of hypertriglyceridaemia: implications for definition, diagnosis, and management. Lancet Diabetes Endocrinol 2:655-666

31. Hovingh GK, Brownlie A, Bisoendial RJ et al (2004) A novel apoA-Imutation (L178P) leads to endothelial dysfunction, increased arterial wall thickness, and premature coronary artery disease. J Am Coll Cardiol 44:1429-1435

32. Humphries SE, Whittall RA, Hubbart CS et al (2006) Genetic causes of familial hypercholesterolaemia in patients in the UK: relation to plasma lipid levels and coronary heart disease risk. J Med Genet 43:943-949

33. Johansen CT, Dube JB, Loyzer MN et al (2014) LipidSeq: a next-generation clinical resequencing panel for monogenic dyslipidemias. J Lipid Res 55:765-772

34. Johansen CT, Hegele RA (2011) Genetic bases of hypertriglyceridemic phenotypes. Curr Opin Lipidol 22:247-253
35. Jones B, Jones EL, Bonney SA et al (2003) Mutations in a Sar1 GTPase of COPII vesicles are associated with lipid absorption disorders. Nat Genet 34:29-31

36. Khera AV, Won HH, Peloso GM et al (2016) Diagnostic yield and clinical utility of sequencing familial hypercholesterolemia genes in patients with severe hypercholesterolemia. J Am Coll Cardiol 67:2578-2589

37. Klose G, Laufs U, März W et al (2014) Familial hypercholesterolemia: developments in diagnosis and treatment. Dtsch Arztebl Int 111:523-529

38. Kostner KM, März W, Kostner GM (2013) When should we measure lipoprotein (a)? Eur Heart J 34:3268-3276

39. Laufs U, Scharnagl H, Halle Metal (2015) Treatment options for statin-associated muscle symptoms. Dtsch Arztebl Int 112:748-755

40. Leren TP, Finborud TH, Manshaus TE et al (2008) Diagnosis of familial hypercholesterolemia in general practice using clinical diagnostic criteria or genetic testing as part of cascade genetic screening. Community Genet 11:26-35

41. Lewis GF, Xiao C, Hegele RA (2015) Hypertriglyceridemia in the genomic era: a new paradigm. Endocr Rev 36:131-147

42. Link E, Parish S, Armitage J et al (2008) SLCO1B1 variants and statin-induced myopathy - a genomewide study. N Engl J Med 359:789-799

43. Ljunggren SA, Levels JH, Hovingh K et al (2015) Lipoprotein profiles in human heterozygote carriers of a functional mutation P297S in scavenger receptor class B1. Biochim Biophys Acta 1851:1587-1595

44. Marks D, Thorogood M, Neil HA et al (2003) A review on the diagnosis, natural history, and treatment of familial hypercholesterolaemia. Atherosclerosis 168:1-14

45. März W, Baumstark MW, Scharnagl H et al (1993) Accumulation of 'small dense' low density lipoproteins in a homozygous patient with familial defective apolipoprotein B-100 results from heterogenous interaction of LDL-subfractions with the LDL receptor.JClin Invest 92:2922-2933

46. März W, Kleber ME, Scharnagl Het al (2017) Clinica importance of HDL cholesterol. Herz 42(1):58-66

47. März W, Ruzicka V, Pohl T et al (1992) Familial defective apolipoprotein B-100: mild hypercholesterolemia without atherosclerosis in a homozygous patient. Lancet 340:1362

48. März W, Scharnagl H, Laufs U (2016) Statinassoziierte Muskelbeschwerden: Mythos oder Wirklichkeit? Herzmedizin 2016:13-19

49. Mignarri A, Magni A, Del Puppo M et al (2016) Evaluation of cholesterol metabolism in cerebrotendinous xanthomatosis. J Inherit Metab Dis 39:75-83

50. Modesto KM, Dispenzieri A, Gertz M et al (2007) Vascular abnormalities in primary amyloidosis. Eu Heart J 28:1019-1024

51. Musunuru K, Pirruccello JP, Do R et al (2010) Exome sequencing, ANGPTL3 mutations, and familial combined hypolipidemia. N Engl J Med 363:2220-2227

52. Nauck MS, Köster W, Dörfer K et al (2001) Identification of recurrent and novel mutations in the LDL receptor gene in German patients with familial hypercholesterolemia. Hum Mutat 18:165-166

53. Nauck MS, Nissen H, Hoffmann MM et al (1998) Detection of mutations in the apolipoprotein CII gene by denaturing gradient gel electrophoresis. Identification of the splice site variant apolipo- protein Cll-Hamburg in a patient with severe hypertriglyceridemia. Clin Chem 44:1388-1396

54. Nauck MS, Scharnagl $\mathrm{H}$, Nissen $\mathrm{H}$ et al (2000) FH-Freiburg: a novel missense mutation (C317Y) in growth factor repeat $A$ of the low density lipoprotein receptor gene in a German patient with homozygous familial hypercholesterolemia. Atherosclerosis 151:525-534

55. Nordestgaard BG, Chapman MJ, Ray K et al (2010) Lipoprotein(a) as a cardiovascular risk factor: current status. Eur Heart J 31:2844-2853

56. Pasanen MK, Neuvonen M, Neuvonen PJ et al (2006) SLCO1B1 polymorphism markedly affects the pharmacokinetics of simvastatin acid. Pharmacogenet Genomics 16:873-879

57. Piepoli MF, Hoes AW, Agewall S et al (2016) 2016 European Guidelines on cardiovascular disease prevention in clinical practice: The Sixth Joint Task Force of the European Society of Cardiology and Other Societies on Cardiovascular Disease Prevention in Clinical Practice (constituted by representatives of 10 societies and by invited experts). Developed with the special contribution of the European Association for Cardiovascular Prevention \& Rehabilitation (EACPR). Eur Heart J 37:2315-2381

58. Putz-Bankuti C, Datz C, März W et al (2006) Clinical-pathological conference series from the Medical University of Graz: case no. 131: elevated transaminases in a 30-year-old male. Wien Klin Wochenschr 118:769-775

59. Reiner Z, Catapano AL, De Backer $G$ et al (2011) ESC/EAS Guidelines for the management of dyslipidaemias: the TaskForce for the management of dyslipidaemias of the European Society of Cardiology (ESC) and the European Atherosclerosis Society (EAS). Eur Heart J 32:1769-1818

60. Reiner Z, Guardamagna O, Nair D et al (2014) Lysosomal acid lipase deficiency - an underrecognized cause of dyslipidaemia and liver dysfunction. Atherosclerosis 235:21-30

61. Ritsch A, Scharnagl H, Eller P et al (2010) Cholesteryl ester transfer protein and mortality in patients undergoing coronary angiography: the Ludwigshafen Risk and Cardiovascular Health study. Circulation 121:366-374

62. Rust S, Rosier M, Funke $\mathrm{H}$ et al (1999) Tangier disease is caused by mutations in the gene encoding ATP-binding cassette transporter 1. Nat Genet 22:352-355

63. Schaaf CP, Koster J, Katsonis P et al (2011) Desmosterolosis-phenotypic and molecular characterization of a third case and review of the literature. Am J Med Genet A 155A:1597-1604

64. Schaefer EJ, Santos RD, Asztalos BF (2010) Marked $\mathrm{HDL}$ deficiency and premature coronary heart disease. Curr Opin Lipidol 21:289-297

65. Silbernagel G, Chapman MJ, Genser B et al (2013) High intestinal cholesterol absorption is associated with cardiovascular disease and risk alleles in ABCG8 and ABO: evidence from the LURIC and YFS cohorts and from a meta-analysis. J Am Coll Cardiol 62:291-299

66. Silbernagel G, Fauler G, Hoffmann MM et al (2010) The associations of cholesterol metabolism and plasma plant sterols with all-cause and cardiovascular mortality. J Lipid Res 51:2384-2393

67. Silbernagel G, Fauler G, Renner W et al (2009) The relationships of cholesterol metabolism and plasma plant sterols with the severity of coronary artery disease. J Lipid Res 50:334-341

68. Soutar AK, Naoumova RP (2007) Mechanisms of disease: genetic causes of familial hyper- 
cholesterolemia. Nat Clin Pract Cardiovasc Med 4:214-225

69. Stitziel NO, Stirrups KE, Masca NG et al (2016) Coding variation in ANGPTL4, LPL, and SVEP1 and the risk of coronary disease. $\mathrm{N}$ Engl J Med 374:1134-1144

70. Stroes ES, Thompson PD, Corsini A et al (2015) Statin-associated muscle symptoms: impact on statin therapy - European Atherosclerosis Society Consensus Panel Statement on Assessment, Aetiology and Management. Eur Heart J 36:1012-1022

71. TalmudPJ, Futema M, Humphries SE (2014) The genetic architecture of the familial hyperlipidaemia syndromes: rare mutations and common variants in multiple genes. Curr Opin Lipidol 25:274-281

72. Talmud PJ, Shah S, Whittall R et al (2013) Use of low-density lipoprotein cholesterol gene score to distinguish patients with polygenic and monogenic familial hypercholesterolaemia: a case-control study. Lancet 381:1293-1301

73. Taylor A, Wang D, Patel K et al (2010) Mutation detection rate and spectrum in familial hypercholesterolaemia patients in the UK pilot cascade project. Clin Genet 77:572-580

74. Teupser D, Baber R, Ceglarek U et al (2010) Genetic regulation of serum phytosterol levels and risk of coronary artery disease. Circ Cardiovasc Genet 3:331-339

75. Umans-Eckenhausen MA, Defesche JC, Van Dam MJ et al (2003) Long-term compliance with lipidlowering medication after genetic screening for familial hypercholesterolemia. Arch Intern Med 163:65-68

76. Vergeer M, Korporaal SJ, Franssen R et al (2011) Genetic variant of the scavenger receptor $\mathrm{Bl}$ in humans. NEngl J Med 364:136-145

77. Versmissen J, Oosterveer DM, Yazdanpanah $M$ et al (2008) Efficacy of statins in familial hypercholesterolaemia: a long term cohort study. BMJ 337:a2423

78. Walma EP, Wiersma TJ (2006) NHG-Standpunt Diagnostiek en behandeling van familiaire hypercholesterolemie. Huisarts Wet 49:202-204

79. Wierzbicka-Rucinska A, Janczyk W, Lugowska A et al (2016) Diagnostic and therapeutic management of children with lysosomal acid lipase deficiency (LAL-D). Review of the literature and own experience. Dev Period Med 20:212-215

80. Wild PS, Zeller T, Schillert A et al (2011) A genome-wide association study identifies LIPA as a susceptibility gene for coronary artery disease. Circ Cardiovasc Genet 4:403-412

81. Young SG, Davies BS, Voss CV et al (2011) GPIHBP1, an endothelial cell transporter for lipoprotein lipase. J Lipid Res 52:1869-1884

82. Zanoni P, Khetarpal SA, Larach DB et al (2016) Rare variant in scavenger receptor $\mathrm{BI}$ raises $\mathrm{HDL}$ cholesterol and increases risk of coronary heart disease. Science 351:1166-1171

83. Zhao Z, Tuakli-Wosornu Y, Lagace TA et al (2006) Molecular characterization of loss-offunction mutations in PCSK9 and identification of a compound heterozygote. Am J Hum Genet 79:514-523

\section{Studie: Auch kurzfristige Steroidtherapie erhöht die Risiken}

Die kurzfristige Verordnung von oralen Steroiden, die aufgrund der raschen Symptomlinderung eine beliebte Therapie auch bei vielen akut entzündlichen Erkrankungen ist, zog in einer retrospektiven Kohortenstudie einen signifikanten Anstieg von Sepsiserkrankungen, venösen Thromboembolien und Knochenbrüchen nach sich.

Die langfristigen Folgen einer Steroidbehandlung sind hinlänglich bekannt. Infektionen, venöse Thromboembolien, avaskuläre Nekrosen und Frakturen gehören ebenso dazu wie Typ-2-Diabetes, arterielle Hypertension und Osteoporose, die das iatrogene Cushing-Syndrom umschreiben. Die kurzfristige Therapie von Steroiden über wenige Tage wird von den meisten Ärzten als sicher betrachtet, obwohl Studien negative Auswirkungen auf die Immunabwehr und den Knochenstoffwechsel dokumentiert haben. Von 1,5 Millionen US-Privatversicherten im Alter von 18 bis 64 Jahren, deren Daten Akbar Waljee von der Universität von Michigan in Ann Arbor und Mitarbeiter auswerteten, hatten 327.452 Versicherte, also mehr als jeder fünfte, innerhalb von drei Jahren mindestens einmal als ambulante Patienten kurzfristig Steroide verschrieben bekommen. Die mediane Dauer der Behandlung betrug sechs Tage und die mediane Dosis $20 \mathrm{mg} /$ die Prednisolon-Äquivalent (die beliebteste Verordnung ist ein "dosepak" mit Methylprednisolon für sechs Tage, das fast der Hälfte der Patienten verordnet wurde). Die Verordnung blieb in den meisten Fällen ohne Folgen. Aber 170 der 327.452 Patienten (0,05 Prozent) erkrankten fünf bis 90 Tage nach der Verordnung an einer Sepsis. Bei 472 Patienten (0,14 Prozent) kam es während der gleichen Zeit zu einer venösen Thromboembolie, 1.657 Patienten (0,51 Prozent) erlitten einen Knochenbruch. Unter den 1.221.493 Versicherten, die keine Steroide erhalten hatten, erkrankten deutlich weniger Patienten an einer dieser drei Erkrankungen.

\section{So wenig und so kurz wie möglich}

Die Forscher verglichen die Inzidenz der drei Komplikationen an den Tagen 5 bis 30 und an den Tagen 31 bis 90 nach Behandlungsbeginn mit einer gleichlangen Phase vor der Verordnung der Steroide. Diese „Self-Controlled-Cases Series"-Methode (SCCS) ergab, dass die Inzidenzrate (IRR) für eine Sepsis im ersten Monat nach der Verordnung des oralen Kurz- zeitsteroids um den Faktor 5,30 (95-ProzentKonfidenzintervall 3,80 bis 7,41 ) signifikant erhöht war. Für die venöse Thromboembolie betrug die IRR $3,33(2,78-3,99)$ und für Knochenbrüche 1,87 (1,69-2,07). Passend zu der Vermutung einer Arzneimittelkomplikation ist, dass die IRR im Zeitraum 31 bis 90 Tage abgeschwächt war. Die Autoren ermitteln eine IRR von 2,91 $(2,05-4,14)$ für die Sepsis, eine IRR von $1,44(1,19-1,74)$ für die venöse Thromboembolie und eine IRR von 1,40 $(1,29-1,53)$ für Knochenbrüche. Auch eine Dosis-Wirkungsbeziehung war ansatzweise erkennbar. Schließlich verglichen die Autoren Patienten mit der gleichen Erkrankung. In jeder Indikationsgruppe kam es bei den Patienten, die orale Steroide erhalten hatten, in den ersten 30 Tagen signifikant häufiger zu Sepsis, venösen Thromboembolien oder Knochenbrüchen.

Obwohl retrospektive Untersuchungen fehleranfällig sind, hat Waljee aufgrund der konsistenten Ergebnisse in den unterschiedlichen Analysen keine Zweifel, dass die Komplikationen Folge der Steroidbehandlung sind. Die Komplikationen waren zwar insgesamt selten, ihre Folgen für die Patienten jedoch gravierend. Die Ärzte sollten deshalb wissen, dass Steroide auch bei einer kurzfristigen Verordnung nicht ohne Risiken sind, meint Waljee. Sie sollten nicht von dem Grundsatz abweichen, stets die niedrigste effektive Dosis für einen möglichst kurzen Zeitraum zu verordnen.

Quelle: Deutsches Ärzteblatt (www.aerzteblatt.de)

basierend auf: BMJ (2017) 357: j1415 